Research Article

\title{
Hybrid Ring-Star-Tree Topology with Optical Add Drop Multiplexer: Cost Effective and Capacity Enhancement
}

\author{
Anju Bala* and Sanjeev Dewra \\ Department of Electronics \& Communication Engineering, Shaheed Bhagat Singh State Technical Campus Ferozepur 152004, Punjab, \\ India.
}

Received 27 July 2016; Accepted 4 June 2017

\begin{abstract}
Optical Add Drop Multiplexer (OADM) has capability to add \& drop the new channels and provides the wavelength routing between the topologies. In this paper, the performance of Ring-Star-Tree hybrid topology with OADM is investigated with $0.8 \mathrm{~nm}$ channel spacing at bit rate of $20 \mathrm{Gbps}$. It is observed that at minimum input transmission power of $-17 \mathrm{dBm}$, hybrid network supports 32 and 128 users for star and tree topology respectively with acceptable Q-Factor. The distance between the nodes of ring network is $150 \mathrm{~km}$ without dispersion compensating fiber.
\end{abstract}

Keywords: Bit Error Rate (BER), Optical Add \& Drop Multiplexer (OADM), Quality Factor (Q-Factor), Semiconductor Optical Amplifier (SOA).

\section{Introduction}

The growth of optical transmission networks is possessed by cost, power dissipation, adaptability and increased capacity [1]. Nowadays, fast evolution of internet service should require more and more network capacity [2]. Due to increase in the demands, operators moves towards optical core networks. This network provides excellent transmission capacity and high bandwidth. The maximum number of users is connected by using the network topologies [3]. Optical network topologies can accommodate high bandwidth, security, efficiency and less transmission cost [4] In the optical fiber, due to less noise data is transmitted with high speed and covers large distance over guided and unguided media. Addition of optical amplifier improves the performance of the optical networks [5].

The demand of new generation of optical networks is high data rate of about tera bit per second. This high data rate can be obtained if the data remain in optical domain and eliminates the requirements of conversion of signals from optical to electronic and back to optical. Therefore, advanced optical networks need all optical ultra signal processing such as wavelength conversion, arithmetic processing and optical logic, add drop function etc. to successfully achieve the required high data rate [6].

It is appropriate to add or drop various wavelengths in optical network system. The OADM is the prominent component to add/drop various or specific wavelength. It improves the connectivity and versatility of the network [7]. OADM contain wavelength multiplexer/demultiplexer, optical switch and channel equalization element. The $2 \times 2$ optical switch achieves wavelength routing between two different routes.

\footnotetext{
*E-mail address: anjurajput18@gmail.com ISSN: 1791-2377 @ 2017 Eastern Macedonia and Thrace Institute of Technology. All rights reserved. doi:10.25103/iestr.103.25
}

OADM provides best use of various features such as network ability, network security, wavelength routing and many more. OADMs based optical metro networks will manage security and translucency in future networks to supply different bit rates to various users [8].

Wavelength Division Multiplexing (WDM) ring networks are designed to reduce the overall cost, which include the cost of electrical components as well as the cost of optical components [9]. A lot of research attention has been attracted by WDM star network as architecture for local/metro area network applications due to its less network cost and simple network operation [10]. Tree network covers broad geological area and increase the ability of system.

Surinder Singh [11] evaluated the performance of ring, bus, star and tree networks at bit rate of $10 \mathrm{Gbps}$ using optimized semiconductor optical amplifiers (SOAs). Ring network supports 27 users and the transmission distance is $1.1 \mathrm{~km}$ with single mode fiber (SMF) and dispersion compensating fiber (DCF) between the nodes. In star network, only 16 users supported.

Hehong Fan et al. [12] designed a star-cross-bus network architecture which is centrally controlled hybrid restoration system with 128 Optical Network Unit. Although centrally controlled, single-distribution fiber at distance $10 \mathrm{~km}$ failure was reestablished. Gurpreet Kaur [13] investigated the Optical Phase Conjugation (OPC) module which is placed in hybrid (Bus-Ring) network topology to suppress or eliminates a number of impairments at $10 \mathrm{Gbps}$.

In this paper, we proposed the hybrid Ring- Star-Tree network topology with OADM. OADM allows the network and which make the system cost effective should be which makes the system cost effective. The previous work [14] was carried for Ring topology supported 60 users using DCF with $126 \mathrm{~km}$ distance between nodes at input power of -10 $\mathrm{dBm}$.

The work is extended here with Ring- Star-Tree hybrid topology at 20 Gbps bit rate with minimum input transmission power from $-17 \mathrm{dBm}$ to $-5 \mathrm{dBm}$ and total 
supported users are 160 at the end of star and tree network. Ring network has $150 \mathrm{~km}$ distance between the nodes without DCF.

This paper is framed as follows. Section 1 presents the introduction. In section 2, the simulation setup of the hybrid network is discussed. Section 3 includes the results and discussion of hybrid network. The Section 4 gives conclusion of the system.

\section{Simulation Setup}

The schematic setup of Ring-Star-Tree hybrid network topology with OADM is shown in Fig.1. A transmitter section consists of PRBS (Pseudo Random Binary Sequence generator), NRZ (Non-Return to Zero) rectangular driver, continuous wave $(\mathrm{CW})$ laser, Mach Zehnder modulator (MZM) as shown in Fig.2. The CW laser array has $-17 \mathrm{dBm}$ initial input power, $10 \mathrm{MHz}$ line width and $0.8 \mathrm{~nm}$ channel spacing. PRBS generates a bit rate of 20 Gbps having alternate operation mode. The outputs of PRBS and CW laser array are given to MZM having extinction ratio of 30 $\mathrm{dB}$. The modulated signal from MZM is given to ring network.

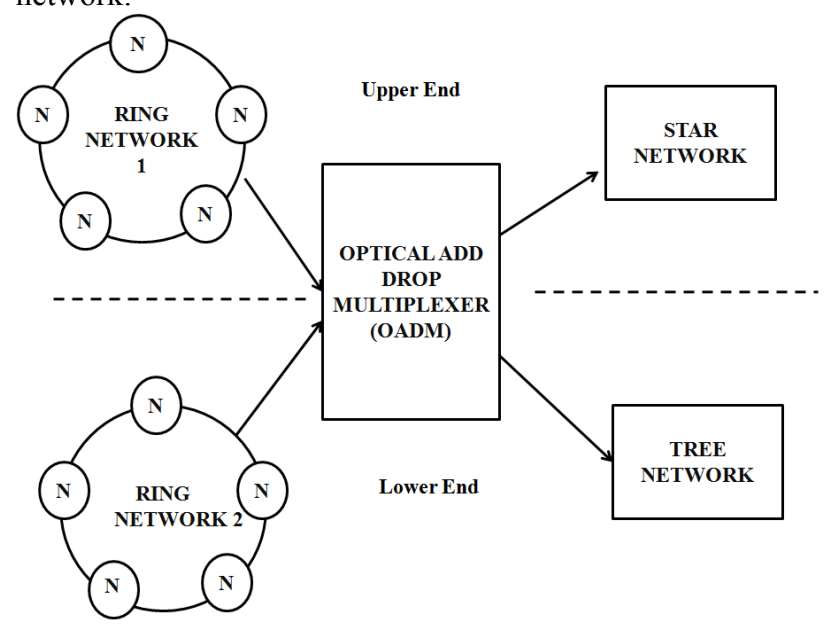

Fig.1. System Setup

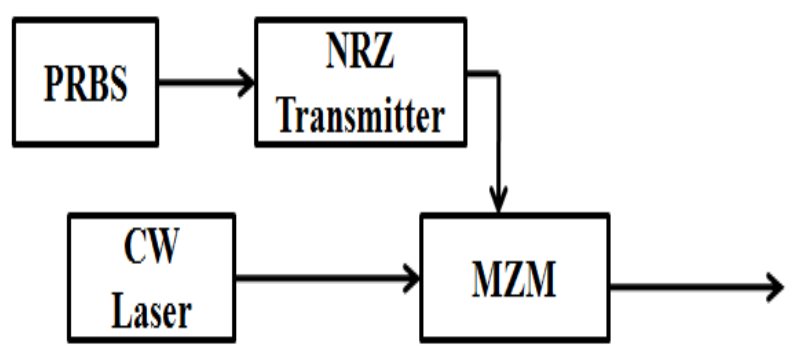

Fig.2. Internal architecture of transmitter section

In ring topology, each node is associated to the other by a single mode fiber (SMF) of length of $150 \mathrm{~km}$ with dispersion of $16 \mathrm{ps} / \mathrm{nm} / \mathrm{km}$ and an optical semiconductor amplifier (SOA) with injection current $0.15 \mathrm{~A}$. Each node is linked through the optical coupler having 0.5 coupling coefficient. Each ring topology contains 32 nodes. The structure of ring network is presented in Fig.3.

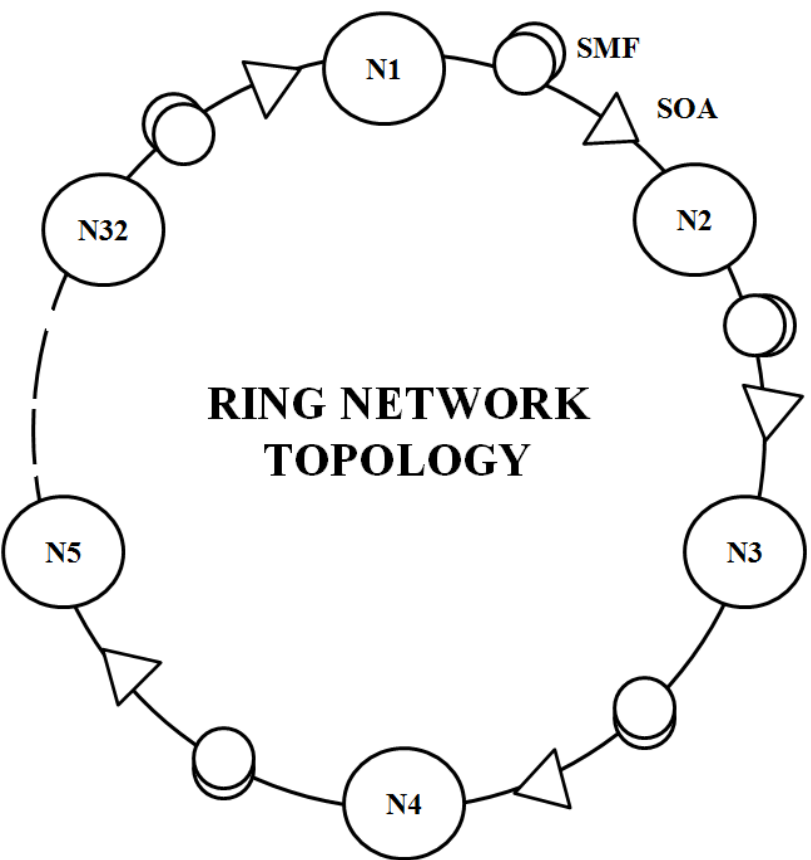

Fig.3. Ring Network Topology

The output from ring topology is fed to OADM. An OADM could be a device to add or drop and routing of various channels carrying wavelength of light in WDM system. It is considered to be a specific type of Optical Cross Connects (OXC).

It has tendency to add/drop different wavelengths and provide greater flexibility to the network. The internal architecture of OADM is shown in Fig.4. The OADM architecture consists of two $1 \times 32$ demultiplexers (Demux 1 , Demux2), two $32 \times 1$ multiplexers (mux1, mux2) and 16 optical switches. The output from ring network is demultiplexed into 64 channels using Demux1 and Demux2 then output from demultiplexers goes to optical switch with parallel input. An optical switch has $\pi$ phase shift. The signals from optical switch are given to mux1 and mux2. The output of mux 1 and mux 2 is given to star topology and tree topology respectively.

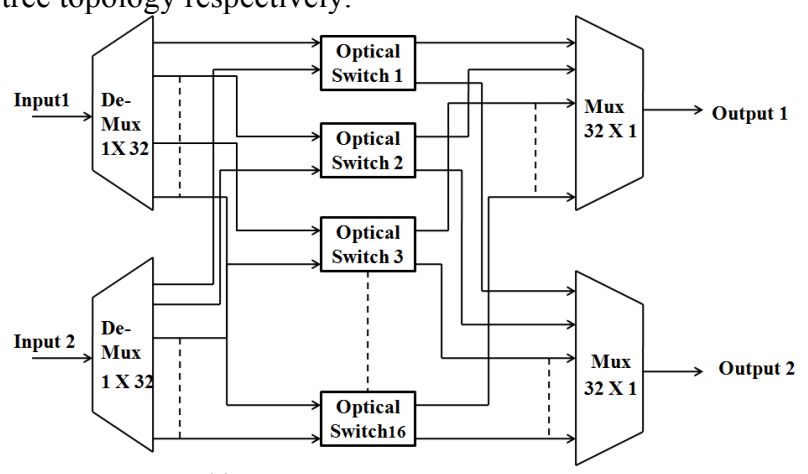

Fig.4. OADM Architecture

In forming star optical network, the $2 \times 2$ optical couplers are used to form $U_{N} \times U_{N}$ network with coupling coefficient of 0.5 to merge or disperse the signal. In star topology, nodes are coupled to a central hub. An $U_{N} \times U_{N}$ star network has $\log _{2} U_{N}$ stages and requires $\left(U_{N} / 2\right) \log _{2} U_{N}, 2 \times 2$ optical couplers; where $U_{N}$ is the total users supported by the star network. The number of users can be increased which enhances the capacity of the system. Star topology supports 32 users as shown in Fig.5. In star topology, transmission 
frequency is dedicated to users and receivers receive the signals through frequency matching.

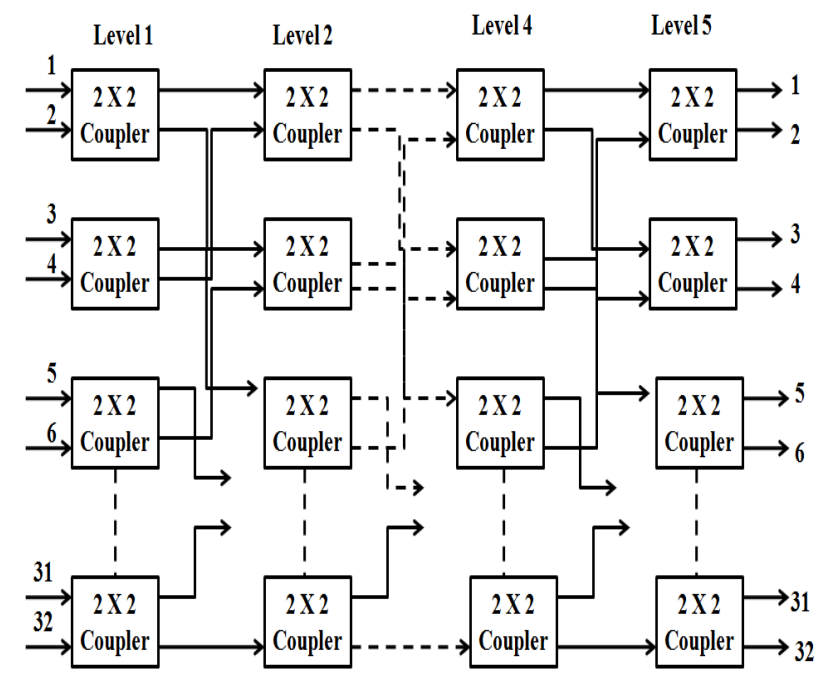

Fig.5. Star Topology

The equation (1) and equation (2) are used to express the number of optical couplers and levels for star topology [11].

Number of optical couplers used $=\left(U_{N} / 2\right) \log _{2} U_{N}$

Number of levels $=\log _{2} U_{N}$

The tree topology is the combination of star and bus topology as shown in Fig.6. Star topology is main topology and bus topology is used as auxiliary topology in tree network. The $2 \times 2$ optical coupler is used at the node of tree topology. Tree topology supports maximum number of users.

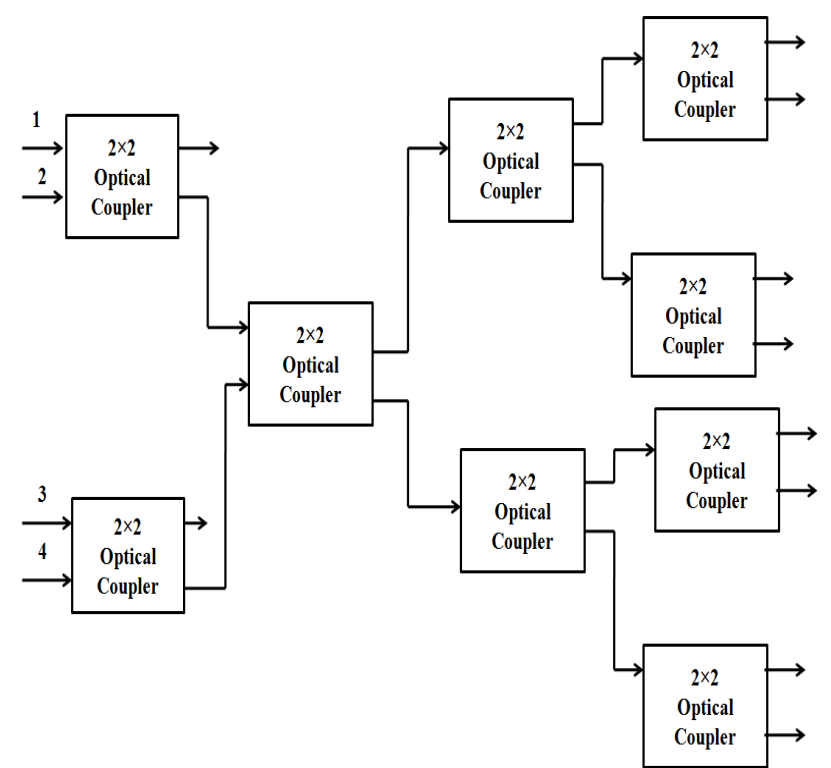

Fig.6. Tree Topology

The receiver consists of Avalanche photo diode (APD), low pass filter, $3 \mathrm{R}$ regenerator and a BER analyzer. APD is used as a photo detector which converts the light signal into electrical signal which is centered at a maximum power with Gain $3 \mathrm{~dB}$, Responsivity $2 \mathrm{~A} / \mathrm{W}$ and ASE noise. The low pass Bessel filter works as an electrical filter with $10 \mathrm{GHz}$ cut off frequency. BER analyzer is used to analyze the quality factor (Q-Factor), Bit error rate (BER) and Eye Height.

\section{Results and Discussion}

In this paper, the performance of Ring-Star-Tree network with OADM has been analyzed in terms of BER, Q-Factor, Received Power and Eye Height with different input powers. The results are obtained at Star and Tree network with 20 Gbps bit rate considering noise at receiver side. Star and tree network support 32 and 128 users respectively. Total users with the combination of Star and Tree network are 160.

The plot of BER versus number of nodes for star and tree topology at different input powers is shown in Fig.7. The variation in BER at nodes with same input power is due to fiber non-linearity, dispersion and loss in the power of signal at the node end. At node 1 , the values of BER are $3.75 \mathrm{e}^{-149}$ for $-5 \mathrm{dBm}, 9.75 \mathrm{e}^{-50}$ for $-10 \mathrm{dBm}, 1.59 \mathrm{e}^{-17}$ for $-15 \mathrm{dBm}$ and $6.67 \mathrm{e}^{-12}$ for $-17 \mathrm{dBm}$. The BER and Q-factor can be predicted as specified in the equation (3) and (4).

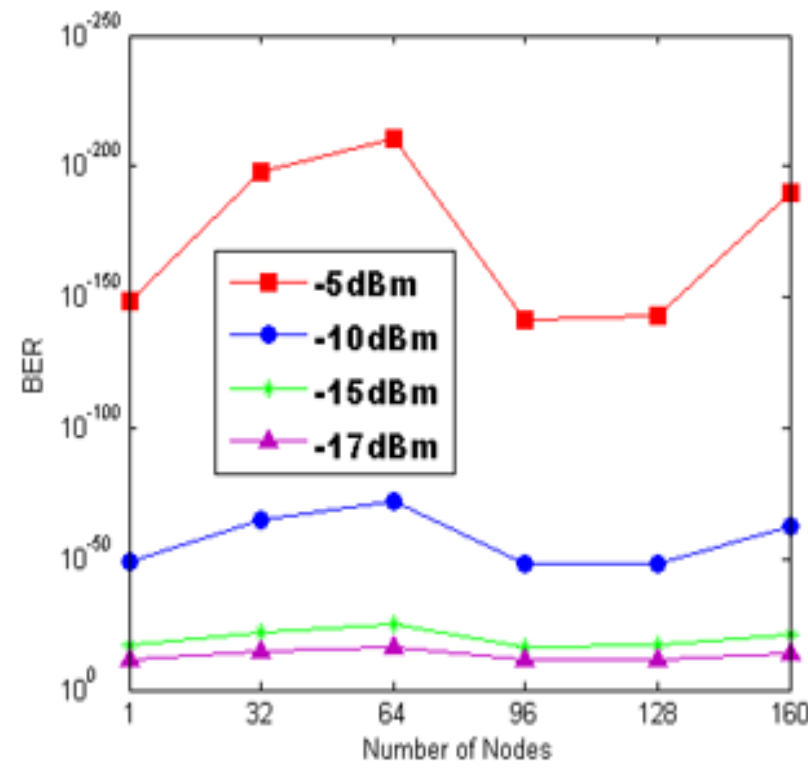

Fig.7. BER versus Number of Nodes

$\mathrm{BER}=\frac{1}{2} \operatorname{erfc}(\mathrm{Q} \mid 2) \approx \frac{\exp \left(-\mathrm{Q}^{2} \mid 2\right)}{\mathrm{Q} \sqrt{2 \pi}}$

Where Q is Q-factor and can be expressed as,

$\mathrm{Q}=\frac{\mathrm{m}_{1}-\mathrm{m}_{0}}{\sigma_{1}-\sigma_{0}}$

where $m_{1}$ and $m_{0}$ represents the mean of the received signal at the sampling instant while bits 1 and 0 are transmitted and $\sigma_{1}, \sigma_{0}$ are the standard deviations, respectively [15]. The Q-factor can be converted into Q-value by taking into decibels.

Fig.8. depicts the variation of Q-Factor with input transmission power at star and tree topology for different nodes at receiver side. It is clear from the graph that with increase in input transmission power from $-17 \mathrm{dBm}$ to -5 $\mathrm{dBm}, \mathrm{Q}-$ Factor increases. It has been analysed that Q-Factor at node 1 is 25.98 for $-5 \mathrm{dBm}, 14.78$ for $-10 \mathrm{dBm}, 8.43$ for $15 \mathrm{dBm}$ and 6.76 for $-17 \mathrm{dBm}$. The variation in Q-Factor at the node end is due to fiber non-linearities, noise at receiver side and loss in the input power. 


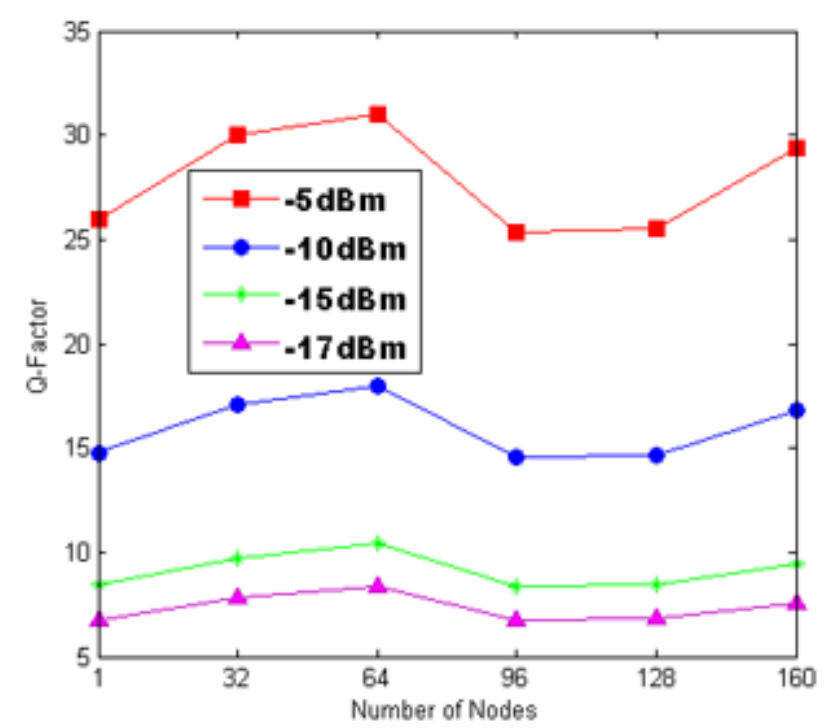

Fig.8. Q-Factor versus Number of Nodes

The variation of received power versus number of nodes at different input transmission power is depicted in Fig.9. The received power at node 1 is $-43.83 \mathrm{dBm}$ for $-5 \mathrm{dBm}$, $48.81 \mathrm{dBm}$ for $-10 \mathrm{dBm},-53.76 \mathrm{dBm}$ for $-15 \mathrm{dBm}$ and $56.15 \mathrm{dBm}$ for $-17 \mathrm{dBm}$. The variation in received power at nodes is due to crosstalk, noise at receiver side and dispersion in fiber. In previous work, results reported for received power is $-70 \mathrm{dBm}$ at input signal power of $-10 \mathrm{dBm}$ whereas in this paper received power is $-56.15 \mathrm{dBm}$ at minimum input transmission power of $-17 \mathrm{dBm}$. The results show an up gradation over the results noted in [14] in terms of distance, users supported, bit rate with minimum input transmission power of $-17 \mathrm{dBm}$.

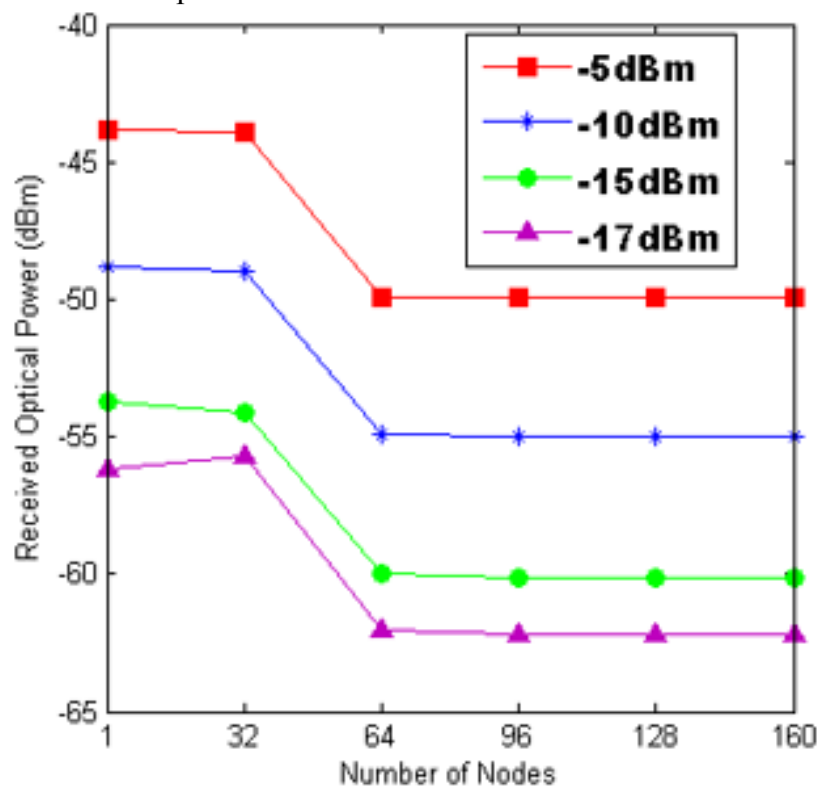

Fig.9. Received Power versus Number of Nodes
Fig.10. shows the variation in Eye Height with respect to input transmission power for star and tree topology. The Eye Height at node 1 is $4.46 \mathrm{e}-007$ for $-5 \mathrm{dBm}, 1.27 \mathrm{e}-007$ for -10 $\mathrm{dBm}, 3.29 \mathrm{e}-008$ for $-15 \mathrm{dBm}$ and $1.80 \mathrm{e}-008$ for $-17 \mathrm{dBm}$. Eye Height determines time delay above which the received signal can be sampled with no error due to interference from adjacent pulses. The height of the eye opening also gives a design about the noise level.

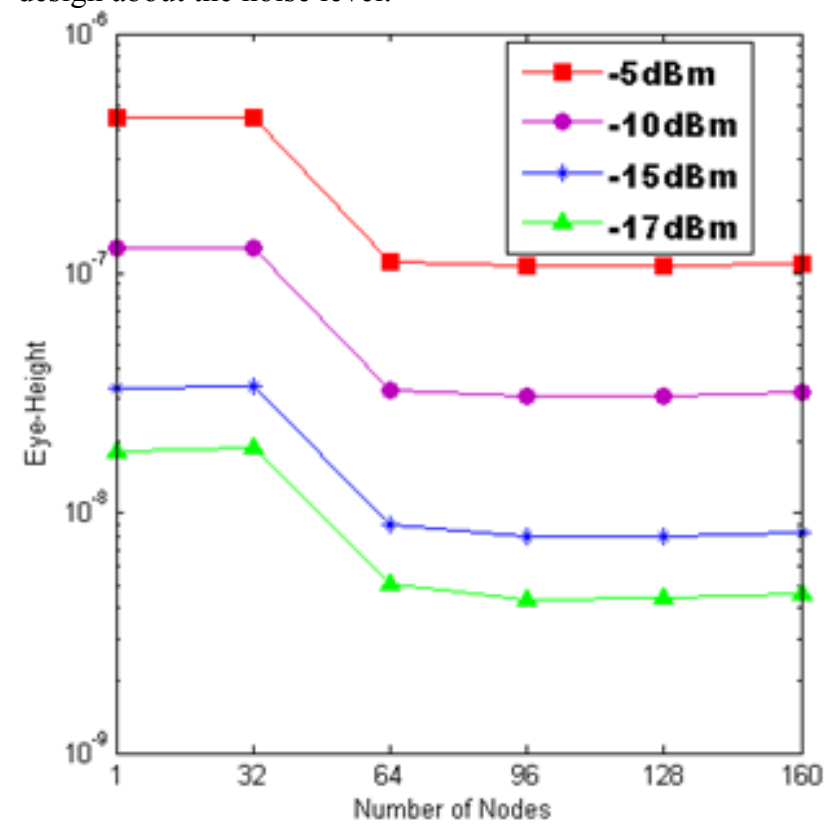

Fig.10. Eye Height versus Number of Nodes

\section{Conclusion}

The feasibility and performance of Ring-Star-Tree hybrid topology with OADM having $0.8 \mathrm{~nm}$ channel spacing at bit rate of $20 \mathrm{Gbps}$ have been demonstrated in terms of BER, QFactor, Received Power and Eye Height. It is observed that hybrid network supports 32 and 128 users for star and tree topology respectively at minimum input transmission power of $-17 \mathrm{dBm}$. The data can be transmitted over a distance of $150 \mathrm{~km}$ without DCF between nodes of ring topology. Moreover, this network is also scalable and cost effective. The complete evaluation of the network is based on hybrid topology with OADM, which proves to be useful for the implementation of hybrid topology as the key foundation in today's infrastructure.

This is an Open Access article distributed under the terms of the Creative Commons Attribution Licence

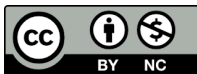

\section{References}

1. A.E.Willner, S.M. Hwant: Transmission of many WDM channels through a cascade of EDFA's in long distance links and ring networks. IEEE J. Lightwave Technology. 13, 802 - 816 (1995)

2. Monika Jain, Soumitra Debnath: Performance of Hybrid Switching Technique for Optical Networks. International Conference on Electrical Engineering/Electronics, Computer, Telecommunications and Information Technology. (1995). doi: 10.1109/ECTICon.2014.6839843
3.S. Singh, R.S. Kaler: Multistage gain-flattened hybrid optical amplifier at reduced wavelength spacing. Optik. 125, 5357-5359 (2014)

4. Sanjeev Dewra, R.S. Kaler: Performance evaluation of an optical network based on optical cross add drop multiplexer. J. Opt. Technology. 80, 502-505 (2013)

5. M. Biswanath Mukherjee: Optical WDM Networks. University of California. Headquarters Location-New York City, pp 15-18. Springer US (2006) 
6. Jitendra Nath Roy: Mach-Zehnder interferometer-based tree architecture for all-optical logic and arithmetic operations. Optik.120, 318-324 (2009)

7. Sanjeev Dewra, R.S. Kaler: Performance analysis of optical network based on optical add drop multiplexers with different MZI techniques. Optik. 124, 347-351 (2013)

8. Yuefeng Jj, Jie Zhang, Yongmei Sun, Wanyi Gu: The Research and Realization of OADM Technology in Metro Optical Network. SPIE, Metro and Access Networks. 4583, 159-170 (2001) doi: $10.1117 / 12.445124$

9. Sun-Sik Roh, Won-Ho So, Young-Chon Kim: Design and Performance Evaluation of Traffic Grooming Algorithms in WDM Multi- Ring Networks. Photonic Network Communications. 3, 335-348 (2001)

10. Yueping Cai, Motoharu Matsuura, Eiji Oki, Naoto Kishi, Tetsuya Miki: A resilient star-ring optical broadcast-and-select network with a centralized multi-carrier light source. Photonic Network Communications. 20, 293-302(2010)
11. Surinder Singh: Performance comparison of optical network topologies in the presence of optimized semiconductor optical amplifiers. Journal Opt. Communication Networking. 1, 313-323 (2009)

12. H. Fan, J. Li, X. Sun: Cost-effective scalable and robust star-crossbus PON architecture using a centrally controlled hybrid restoration mechanism. J. Opt. Communication Networking. 5,730-740 (2013)

13. Gurpreet Kaur, M.S. Patterh, Ramandeep Kaur: Four wave mixing power suppression in hybrid network topology using optical phase conjugation module. Optik. 126, 347-349 (2015)

14. Gurpreet Kaur, M.S.Patterh: Performance investigation of ring network topology in the presence of semiconductor optical amplifiers. Optik. 124, 5366-5368 (2013)

15. Simranjit Singh, Amit Kapoor, Gurpreet Kaur, R.S. Kaler, Rakesh Goyal: Investigation on wavelength re-modulated bi-directional passive optical network for different modulation formats. Optik. $125,5378-5382$ (2014) 